\title{
Effects of Overspeed or Overload Plyometric Training on Jump Height and Lifting Velocity
}

\section{(ㄷ) (1) (우 $\Theta$}

\author{
Authors \\ Nicolay Stien, Morten Strate, Vidar Andersen, Atle Hole Saeterbakken
}

\begin{abstract}
Affiliation
Key words

$\begin{array}{ll}\text { received } & 03.10 .2019 \\ \text { revised } & 07.01 .2020 \\ \text { accepted } & 09.02 .2020\end{array}$
\end{abstract}

Department of Sport, Food and Natural Sciences, Hogskulen pa Vestlandet - Campus Sogndal, Sogndal, Norway

velocity, training, muscle thickness, testing

Bibliography

DOI https://doi.org/10.1055/a-1116-0749

Sports Medicine International Open 2020; 4: E32-E38

(c) Georg Thieme Verlag KG Stuttgart · New York

ISSN 2367-1890

\section{Correspondence}

Dr. Atle Sæterbakken

Department of Sport, Food and Natural Sciences, Hogskulen pa Vestlandet - Campus Sogndal

6856 Sogndal

Norway

Tel.: + 475667000

atle.saeterbakken@hvl.no

\section{ABSTRACT}

The aim of this study was to examine the effects of overspeed or overload plyometric training on jump height and lifting velocity in resistance trained females without plyometric training experience. Fifty-six participants (age: $21.2 \pm 1.7$ years; body mass: $65.1 \pm 8.2 \mathrm{~kg}$; height: $168.0 \pm 5.9 \mathrm{~cm})$ were randomly allocated to either an overspeed ( $n=18)$, overload $(n=18)$, or passive control $(n=16)$ group. The two training groups completed $18.7 \pm 1.7$ sessions consisting of three different plyometric exercises with overspeed or overload over eight weeks. Apart from the external loading, the two training modalities were identical. Following the training period, the changes in the recorded variables were not significantly different from those in the control group, nor did the training groups differ from each other. The training groups improved peak and average lifting velocity in the 40 and $60 \%$ of body mass loading conditions $(9.50-33.37 \%, p=<0.001-0.038)$, whereas only the average lifting velocity improved in the $80 \%$ of body mass loading condition (OS: $14.47 \%, \mathrm{p}<0.001$ and OL: $23.13 \%$, $\mathrm{p}<0.001)$. No significant changes occurred in the control group (9.18-13.55\%, P = 0.062-0.980). Overspeed and overload plyometric training may be viable methods for improving lifting velocity, but not squat jump height, in a population without plyometric training experience.

\section{Introduction}

Plyometric training (PT) is a form of high-velocity training utilizing the stretch-shortening cycle in a rapid eccentric-concentric muscle action [1]. PT is typically used to improve power output (force $\times$ velocity) in high-velocity movements, such as jumping [1-3]. PT utilizing overload (OL) and overspeed (OS) has recently gained increased attention [4-8]. OL training involves adding additional load to an individual's body mass (BM) and therefore increasing the force component of the force-velocity curve, whereas OS focuses on the velocity component by reducing BM [7,9]. Currently, understanding of the adaptations specific to each of these training modalities is limited.

Both OL and OS training have proven more effective than PT utilizing only BM for increasing jump height in participants with considerable experience with high-velocity movements $[4,8,10,11]$. However, few studies have compared the effects of OL and OS [5-7] and these studies have been unable to identify differences in jump height between the OS and OL groups, possibly due to the small differences in applied loads between the groups [6]. However, Markovic et al. [7] speculated that OS training could be more effective for improving jump height, because only the OS group increased jump height more than the control group. Furthermore, Argus et al. [5] found that OS jumps allowed for a higher peak power output than OL jumps.

Of the comparable studies examining the changes in explosive features following PT with OS or OL [5-7], only Markovic et al. [6] included testing of jumps both utilizing the stretch-shortening cycle (counter-movement jumps (CMJ)) and jumps consisting only of a concentric phase (squat jumps (SJ)). The authors reported similar improvements in both jump conditions following both OS and OL training, but the improved CMJ height was not comparable with 
the changes in power output (force $\times$ velocity). Thus, the authors speculated that changes in the movement pattern during the CM] could confound the relationship between jump height and the recorded force and velocity. In order to examine the potential changes in velocity following PT, concentric testing may be better suited.

Although improvements in explosive parameters have been achieved following $O S$ and $O L$ training ranging from $\pm 0.3 \mathrm{BM}$, whether OS or OL training can yield the greatest improvements in jump height and lifting velocity is still uncertain $[4-7,10,11]$. Furthermore, comparable studies have implemented only vertical bilateral jumps [5-7]. Implementing larger loads and a variety of exercises could possibly cause different outcomes. The aim of the present study was, therefore, to compare the effects of a PT program with larger differences in OS and OL using several exercises (bilateral, unilateral, and horizontal jumps), SJ height, and lifting velocity in resistance-trained females without PT experience. Measures of muscle thickness (MT) of the vastus lateralis were also included to help explain the results. Based on previous findings [5, 7], the present study hypothesized that both groups would produce increased SJ height and lifting velocity, but that the OS group would improve more than the $\mathrm{OL}$ group. As a result of the additional load, the OL group was expected to increase MT more than the OS group.

\section{Materials and Methods}

\section{Participants}

For this study, 56 resistance-trained female students aged 19-26 years volunteered to participate ( $\triangleright$ Table 1 ). All eligible volunteers had no illnesses or injuries and had no previous experience with PT in the last six months. After pre-testing, the participants were randomly assigned to one of the following three groups: OS $(n=18)$, OL $(n=18)$, or control (CON; $n=16)$. Due to (1) injuries not related to the intervention, (2) failure to attend the required number of training sessions ( $\geq 16$ of 20 ), or (3) personal reasons, four participants were excluded from further analyses ( $>$ Fig. 1). All participants were informed about the study orally and in writing and signed an informed consent form prior to data collection. The present procedures were performed in accordance with the Declaration of Helsinki and approved by the Norwegian Centre for Research Data $(56489 / 3 / H J T)$. The research was also conducted ethically according to international standards as described for the International Journal of Sports Medicine [12].

\section{Experimental design}

A randomized controlled trial with two training groups and one control group was performed to examine the possible effects on $\mathrm{S}$ ] height, $V_{\text {avg }}$ and $V_{\text {peak }}$ after 20 sessions of PT with OS or OL over eight weeks. The recorded change scores for average S] height $\left(\mathrm{V}_{\text {avg }}\right)$, peak lifting velocity $\left(\mathrm{V}_{\text {peak }}\right)$, and muscle thickness (MT) of the lower limb muscles were used in the analyses.

\section{Procedures}

The tests were performed on two separate days. Anthropometric measurements were conducted on the first day, and physical tests were conducted on the second day. All participants performed the jump test before the lifting velocity measurements. Because the study population had resistance training experience, no familiarization session was performed. Instead, several attempts were given to ensure the highest possible performance in each test. The participants agreed to abstain from leg training $48 \mathrm{~h}$ before testing. The participants were also instructed to not alter their normal activities during the intervention period (controlled via a questionnaire pre- and post-intervention).

\section{Measurements}

Ultrasound imaging (LogicScan 128EXT-1Z; Telemed, Vilnius, Lithuania) was used to measure the thickness of the vastus lateralis muscle on the dominant side. Measurements were taken with the participants in a relaxed and supine position with a bolster under

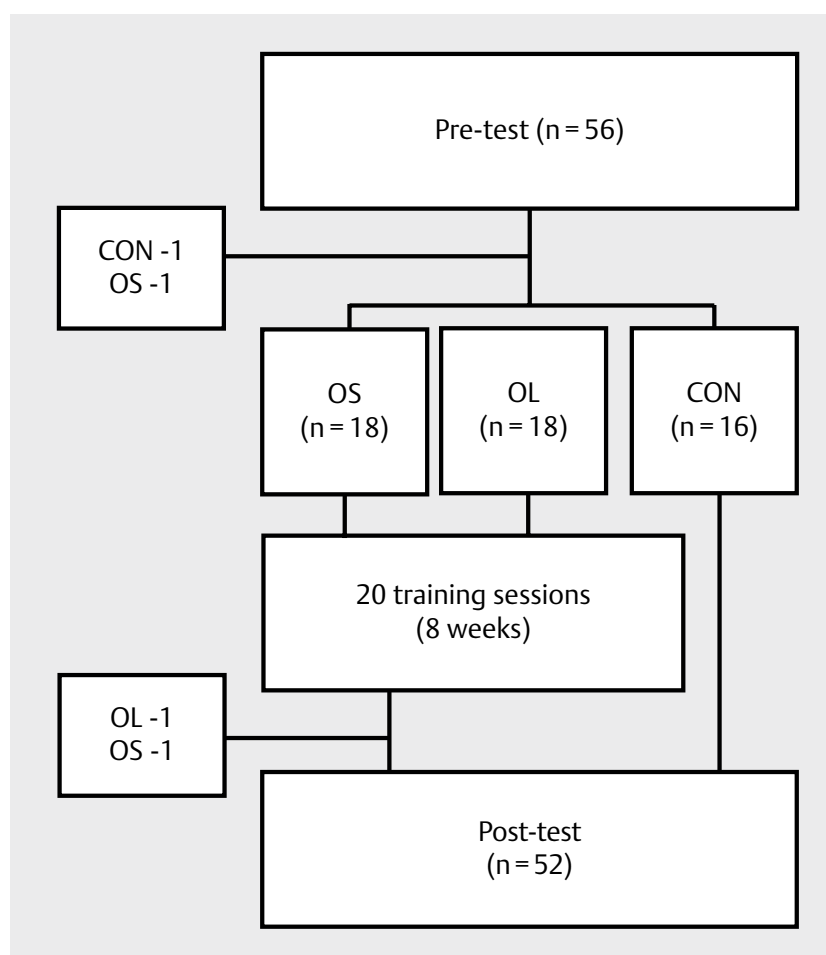

- Fig. 1 Flow chart showing group allocation, pre- and post-test.

- Table 1 Anthropometric characteristics and weekly activity level of the study population.

\begin{tabular}{|l|l|l|l|l|l|l|}
\hline & Age (years) & Height $(\mathbf{c m})$ & Body mass $\mathbf{( k g )}$ & Leg strength training (h) & Endurance training (h) & Other training (h) \\
\hline CON & $21.3(1.8)$ & $169.7(5.3)$ & $69.4(8.3)^{*}$ & $1.5(1.2)$ & $2.9(3.7)$ & $3.8(5.5)$ \\
\hline OS & $21.3(1.3)$ & $167.3(6.8)$ & $62.0(6.8)$ & $1.3(1.3)$ & $1.3(1.3)$ & $2.4(2.8)$ \\
\hline OL & $20.9(1.8)$ & $167.0(5.3)$ & $64.3(7.3)$ & $0.8(1.4)$ & $2.2(2.0)$ & $3.2(3.4)$ \\
\hline \multicolumn{6}{|l|}{ Results are given as mean (SD). h= hours. ${ }^{*}=$ Significantly different from OS (p<0.05). } \\
\hline
\end{tabular}


the knee creating an approximately $170^{\circ}$ knee joint angle $\left(180^{\circ}=\right.$ full extension). The measurements for all participants were taken midway between the lateral condyle and the greater trochanter of the femur [13]. The distance between the anatomical landmarks was recorded to ensure reliability from pre- to post-test. MT was measured (in $\mathrm{mm}$ ) between the superficial and deep connective tissue using the software EchoWavell (Telemed). Five images were taken [14], and the mean value of the three middlemost measurements was used in the analyses ( $C V=1.16-1.19 \%)$.

A standardized warm-up consisting of five CMJ and five SJ was performed before jump testing. Following three minutes rest, $S$ ] height was determined using a force plate (Ergotest Innovation A/S, Porsgrunn, Norway), which was calibrated in accordance with the manufacturer's specifications before testing. SJ were carried out from a $90^{\circ}$ knee angle with the hands fixed at the hip [6]. Jump height was determined by the relative impulse created prior to take-off [15]. The participants performed at least three jumps, but if the last jump was the highest, testing continued until a reduction in performance was observed ( $C V=5.98-7.61 \%)$. The investigators considered an attempt successful if participants' hands remained in contact with the hip throughout the jump, whereas the MuscleLab software (v.10.4.37.4073, Ergotest Innovation A/S) automatically omitted an attempt if any countermovement was detected before the concentric phase. A rest period of at least one minute was given between each attempt, and the best result was used in the analyses [6].

Lifting velocity was measured approximately five minutes after the jump test with no further warm-up. Measures were taken during single repetitions of concentric back-squats with increasing loads. This test was carried out on a Smith machine (Pivot680L; Pivot Fitness, Tianjin, China) to eliminate the stretch-shortening cycle and reduce the technical demands [16]. The barbell height was adjusted so each participant started from a $90^{\circ}$ knee angle. The individual barbell height and foot placement were identical at pre- and post-test. The participants performed one single repetition at $40 \%$ $\mathrm{BM}$ with maximal effort and rested one minute before the load was increased to $60 \% \mathrm{BM}$. Each participant performed three lifts $(40,60$, and $80 \% \mathrm{BM})$. The peak $\left(\mathrm{V}_{\text {peak }}\right)$ and average velocity $\left(\mathrm{V}_{\text {avg }}\right)$ were measured via a linear encoder (Ergotest Innovation $A / S$ ), and registered by the MuscleLab software (v.10.4.37.4073; Ergotest Innovation A/S).

\section{Training}

Elastic rubber bands were used to pull participants upward (OS) or downward (OL), thus mimicking a decrease or an increase in BM without altering body inertia [17]. The applied loading (OL) or unloading (OS) was of equal magnitude, but in opposite directions for the training groups. The bands were anchored either above (OS) or below (OL) the participants via static chains ( $\mathbf{F i g}$. 2) and each participant was given one specific link (based on body height) where the bands should be attached, thus generating the desired load. For the broad jumps, lines were drawn on the floor to indicate where the participants should stand to achieve the planned OS or OL. In the vertical jumps, the bands were attached under the arms through a sling-type harness for the OS group ( $\triangleright$ Figs. $2 \mathbf{2 a}-\mathbf{c}$ ) and at the waist through a modified climbing harness for the OL group ( $\triangleright$ Figs. 2d-f). For the broad jumps, the bands were placed around the waist.
On two occasions during the intervention period (weeks 1 and 5), the maximal force applied by the elastic bands (the mean value of five consecutive jumps) was measured for each participant to ensure that the load $(N)$ was in accordance with the planned training volume ( $>$ Table 2 ). This was done by replacing a portion of the loading apparatus with a force sensor (Ergotest Innovation A/S) while the participants performed one set. The measurements were taken in the lowest squat position for the OS group and right before the feet left the ground for the OL group, using the linear encoder and force sensor synchronized with the MuscleLab system (see www.ergotest.com for additional information). For the $\mathrm{OL}$ group, the take-off moment was identified prior to the measurement. The force data were then collected at that point in the movement. The reported band force is the mean of five consecutive jumps. The applied load for the broad jump exercise was not measured in vivo, but was adjusted to yield the same OS or OL in the takeoff phase as the one-legged jumps (100-150N).

The PT was performed two to three times per week over an eightweek period ( 20 sessions). Participants had to attend $\geq 80 \%$ of the sessions to be included in the analyses and a mean attendance of $93.5 \%$ was reached. Every session was supervised to ensure the highest possible effort and correct execution of the exercises. Before each session, a standardized warm-up procedure was performed. The warm-up consisted of two sets of $10 \mathrm{BM}$ squats followed by two sets of five CMJs with no external load [5]. Each training session consisted of three exercises: two-legged CMJs, one-legged CMJs, and broad jumps. The volume throughout the intervention is presented in - Table 3. All jumps within one set were performed consecutively without pause and at a $90^{\circ} \mathrm{knee}$ angle in the bottom position in both conditions. For the broad jumps, participants immediately moved back to the starting position after landing to perform the next horizontal CMJ, thus ensuring identical band resistance in all jumps with minimal pause between jumps. The participants were given three minutes to rest between each set [5]. The control group did not participate in the training and were asked to continue their normal activities but to avoid systematic jump training.

\section{Statistical analyses}

Statistical analyses were performed using SPSS v.25 (SPSS Inc., Chicago, Illinois, USA). Because SJ height at pre-test demonstrated deviations from normality (Kolmogorov-Smirnov test, $p=0.013$ ), all data were log-transformed to reduce non-uniformity bias. Following log transformation, the data were normally distributed $(p=0.095-0.200)$. The effects were then back-transformed to percent changes. A mixed-model analysis of variance (3 groups $\times 2$ measuring times) was used to examine the differences in the measured variables. When significant interactions of main effects were found, Bonferroni post hoc tests were used to determine where the differences lay. Statistical significance was accepted at $p \leq 0.05$. The data are presented as means with standard deviations (SD) and Cohen's d effect size (ES). The ES was classified as follows: trivial, <0.2; small, 0.2-0.5; medium, 0.5-0.8; and large, >0.8 [18].

\section{Results}

Except for the difference in BM between the OS and CON groups $(p=0.006)$, the three groups were homogenous at baseline $(p=$ 



- Fig. 2 Exercises performed with OS (a, b, and $\mathbf{c}$ ) and OL (d, e, and f). Two-legged CMJ (a and $\mathbf{d})$, one-legged CMJ (b and e) and broad jump (c and f).

- Table 2 Maximal force $(\mathrm{N})$ exerted by the elastic bands during the oneand two-legged CMJ.

\begin{tabular}{|l|l|l|l|l|}
\hline \multirow{2}{*}{} & \multicolumn{2}{|c|}{ One-legged CMJ } & \multicolumn{2}{c|}{ Two-legged CMJ } \\
\cline { 2 - 5 } & OS & OL & OS & OL \\
\hline $\begin{array}{l}\text { Sessions } \\
1-10(\mathrm{~N})\end{array}$ & $-102(10)$ & $100(7)$ & $-217(18)$ & $199(8)^{*}$ \\
\hline $\begin{array}{l}\text { Sessions } \\
11-20(\mathrm{~N})\end{array}$ & $-153(10)$ & $152(5)$ & $-318(20)$ & $298(10)^{*}$ \\
\hline
\end{tabular}

Results are given as mean (SD). $\mathrm{N}=$ newton. ${ }^{*}=$ Significantly different from OS $(p<0.05)$.
$0.138-0.613)$. As BM varied at pre-test, all values were analyzed both relative to $\mathrm{BM}$ and as absolute values. Because the results were identical, only absolute values are presented herein.

S) height demonstrated no group $\times$ time interaction $(F=2.433$, $p=0.098)$ or main effect of group $(F=1.475, p=0.239)$, but a significant main effect of time was observed $(F=30.606, p<0.001)$.

A group $x$ time interaction was found for both velocity measures at all loads $(F=3.733-7.167, p<0.000-0.031)$ except for $V_{\text {peak }}$ in the $80 \%$ BM condition $(F=1.035, p=0.363)$. No differences between the groups were found in either measure in any of the conditions ( $\mathrm{p}=0.416-1.000$; $\vee$ Table 4 ). 
- Table 3 Progression in volume throughout the training period.

\begin{tabular}{|c|l|l|l|l|l|l|}
\hline Week & Sets per exercise & Jumps per set & Number of exercises & Weekly sessions & Jumps per session & Jumps per week \\
\hline $1-2$ & 4 & 5 & 3 & 2 & 60 & 120 \\
\hline $3-4$ & 5 & 5 & 3 & 3 & 75 & 225 \\
\hline $5-6$ & 4 & 5 & 3 & 2 & 60 & 120 \\
\hline $7-8$ & 5 & 5 & 3 & 75 & 225 \\
\hline
\end{tabular}

> Table 4 Pre- and post-test values for peak $\left(V_{\text {peak }}\right)$ and average $\left(V_{\text {avg }}\right)$ velocity $\left(\mathrm{m}^{*} \mathrm{~s}^{-1}\right)$ during concentric back squats in the 40,60 and $80 \%$ of body mass conditions. The results are presented with the relative change (\%) and Cohen's $d$ effect size with their respective p-values.

\begin{tabular}{|c|c|c|c|c|c|}
\hline & Pre- test & Post- test & Percent change & Effect size & $p$ value \\
\hline \multicolumn{6}{|c|}{$\mathrm{V}_{\mathrm{avg}} 40 \%$} \\
\hline CON & $0.769(0.184)$ & $0.846(0.153)$ & $13.55(23.54)$ & 0.46 & 0.062 \\
\hline OS & $0.806(0.138)$ & $0.877(0.125)$ & $10.13(12.28)$ & 0.55 & 0.002 \\
\hline $\mathrm{OL}$ & $0.751(0.118)$ & $0.946(0.082)$ & $28.84(23.34)$ & 1.95 & $<0.001$ \\
\hline \multicolumn{6}{|c|}{$V_{\text {peak }} 40 \%$} \\
\hline CON & $1.577(0.379)$ & $1.657(0.287)$ & $9.18(25.29)$ & 0.24 & 0.432 \\
\hline OS & $1.585(0.312)$ & $1.713(0.261)$ & $10.04(15.46)$ & 0.45 & 0.030 \\
\hline $\mathrm{OL}$ & $1.511(0.253)$ & $1.866(0.197)$ & $26.50(23.20)$ & 1.58 & $<0.001$ \\
\hline \multicolumn{6}{|c|}{$\mathrm{v}_{\mathrm{avg}} 60 \%$} \\
\hline CON & $0.687(0.183)$ & $0.739(0.144)$ & $11.91(25.34)$ & 0.32 & 0.288 \\
\hline OS & $0.712(0.150)$ & $0.771(0.142)$ & $9.50(12.86)$ & 0.40 & 0.012 \\
\hline $\mathrm{OL}$ & $0.676(0.100)$ & $0.808(0.088)$ & $20.62(9.95)$ & 1.41 & $<0.001$ \\
\hline \multicolumn{6}{|c|}{$v_{\text {peak }} 60 \%$} \\
\hline CON & $1.436(0.379)$ & $1.493(0.261)$ & $10.34(32.59)$ & 0.18 & 0.980 \\
\hline OS & $1.401(0.317)$ & $1.513(0.297)$ & $9.65(14.42)$ & 0.36 & 0.038 \\
\hline $\mathrm{OL}$ & $1.344(0.363)$ & $1.666(0.180)$ & $33.37(42.35)$ & 1.18 & $<0.001$ \\
\hline \multicolumn{6}{|c|}{$\mathrm{V}_{\mathrm{avg}} 80 \%$} \\
\hline CON & $0.584(0.153)$ & $0.642(0.152)$ & $12.47(19.23)$ & 0.38 & 0.082 \\
\hline OS & $0.608(0.156)$ & $0.681(0.121)$ & $14.47(12.51)$ & 0.52 & $<0.001$ \\
\hline $\mathrm{OL}$ & $0.578(0.125)$ & $0.701(0.112)$ & $23.13(13.42)$ & 1.03 & $<0.001$ \\
\hline \multicolumn{6}{|c|}{$V_{\text {peak }} 80 \%$} \\
\hline CON & $1.167(0.230)$ & $1.343(0.271)$ & 21.55 (41.94) & - & n.s. \\
\hline OS & $1.258(0.271)$ & $1.367(0.230)$ & $9.84(9.70)$ & - & n.s. \\
\hline $\mathrm{OL}$ & $1.272(0.237)$ & $1.481(0.225)$ & $18.05(14.70)$ & - & n.s. \\
\hline
\end{tabular}

A significant group $\times$ time interaction $(F=4.421, p=0.017)$ was observed for MT. The OS and OL groups increased MT (7.08 $\pm 6.22 \%$, $E S=0.53, p=0.012$ and $8.82 \pm 6.32 \%, E S=0.86$, $p<0.001$ ), whereas MT remained unchanged in the CON group $(1.55 \pm 6.22 \%$, $E S=0.12, p=0.778)$. Still, post hoc tests showed no differences between the groups $(p=0.720-1.000)$.

\section{Discussion}

In contrast to the hypothesis, the differences in SJ height and lifting velocity between the groups did not reach statistical significance. The findings are consistent with comparable studies in which explosive features have improved following PT, albeit not differently between the training groups [5-7]. In contrast with comparable studies, however, the present findings revealed no difference between the outcomes following the training and the control condition. Several reasons may explain the lack of differences between the groups following PT interventions with OS and OL. Importantly, the present study and comparable investigations have included participants without PT experience [5-7]. Due to the novel training stimulus, it is possible that both groups achieved improvements in explosive parameters regardless of the external loads. Regarding the lack of difference between the training groups and the CON group, it is possible that the outside activities performed by the CON group or a learning effect from the pre-test produced nonsignificant improvements in SJ height and lifting velocity that were not different from those achieved following the intervention.

Although the external load for the two-legged CMJ during the second half of the training period was approximately $45 \%$ of the participants' mean BM $(30 \mathrm{~kg})$, the results were comparable to those observed following lower loads ( $30 \%$ of BM) [5-7]. This observation supports the findings of Behm and Sale [19], who concluded that the intended training velocity might determine the velocity-specific adaptations, regardless of the achieved velocity in 
training as dictated by the external load. As the participants were instructed to execute the PT with maximal velocity, the difference in external loads might not have been sufficient to produce different changes in lifting velocity and S] height. Furthermore, some evidence has suggested that the individual force-velocity profile during jumping dictates which training method is more suited for improving jump height and power output [9]. As the group allocation in the present study was randomized, differences in training responses within the training groups may have equalized the training outcomes through large inter-individual variations.

In contrast to the hypothesis, the OS stimulus did not produce higher lifting velocities than the OL training or the control condition. Training with an overspeed stimulus likely made it difficult to develop higher velocity when testing with an extra load. In fact, the observed effect sizes for the OL group (0.91-1.95) were considerably larger than those observed following the OS training (0.360.55 ) and the control condition (0.18-0.62). Interestingly, the effect sizes for the changes in $V_{\text {peak }}$ and $V_{\text {avg }}$ for the OL group increased with decreasing loads, possibly suggesting more meaningful improvement at the load closest to the training load. Because this test only included loading beyond BM, the OL training was likely more specific toward this test [20]. For example, Markovic et al. [7] found differences for jumping velocity between $O S$ and $O L$ only when testing with loads lighter than BM ( $-0.3 \mathrm{BM})$. In contrast to the findings of Markovic et al. [7], however, the current findings suggest that the OS stimulus is also able to improve lifting velocity using external loads. Importantly, Markovic et al. [7] recorded velocity during the concentric phase of CMJ, whereas the present study measured the lifting velocity during concentric back squats. It is possible that changes in movement pattern and utilization of the stretch-shortening cycle are more load-specific, whereas muscular changes are more transferrable across the load spectrum.

To the authors' knowledge, the present study was the first to measure changes in MT after PT with OS or OL. Although both training groups increased MT, the changes were not different between the three groups. The lack of difference from the CON group could be attributed to the relatively low training volume and minor loads used in the intervention. Albeit none of the groups reported changing their outside physical activity, it is possible that the $1.5 \mathrm{~h}$ of weekly leg strength training produced a non-significant increase in MT in the CON group after eight weeks. Inter-individual differences in outside activity volume could also have affected the results. Regarding the similar results between the training groups, one potential reason could be that the muscle fiber type with the largest potential for hypertrophy (type II) is utilized in both forceful (OL) and rapid (OS) contractions [21]. Contrastingly, Bogdanis et al. [22] observed no change in fiber type distribution following increased cross-sectional area after a period of high-velocity eccentric training. The increased MT might have suggested that the improvements in SJ height and lifting velocity were mediated partly by morphological changes. However, architectural changes or electromyography were not measured but should be included in future studies.

Although the present findings may provide additional knowledge about $\mathrm{OL}$ and $\mathrm{OS}$ training, potential limitations of the study should be considered. First, only resistance-trained females without experience with PT were recruited. The results, therefore, may lack generalizability to other populations. Furthermore, unloading the BM in OS training reduces the total training volume. Therefore, the OS group should possibly have performed more sets to compensate for the lower training load. However, owing to individual $\mathrm{BM}$ and leg strength, the relative intensity would have been nearly impossible to control. Because this study aimed to compare training methods with only the external load as the independent variable, changing other factors (e.g., workout duration and number of sets) would complicate the findings. Finally, physical activity or training outside the PT intervention was controlled but not restricted. The self-reported activity level did not differ between the groups, nor did the activity level or type of activities change from pre- to post-test. However, it is possible that the CON group maintained a higher outside activity level than the training groups that was not reported, thereby achieving some non-significant improvements in SJ height and lifting velocity.

Explosive features of the leg extensors are critical factors for performance in several sports. Limited literature exists on PT with OS or OL, and the literature that does exist has not been able to identify clear differences between the training outcomes [5-7]. The current findings suggested that higher lifting velocities at positive loads might be achievable following a plyometric training program with either an overspeed or an overload stimulus, whereas no effect for squat jump height could be identified. A novel finding of the present study was that both training modalities also increased MT similarly across groups. However, none of the tested variables increased more in the training groups compared to the control condition. Future research should examine the effects of PT with greater differences between the loads in the OS and OL groups over a longer period. Including a population with PT experience and a larger sample size could also detect distinct differences between the two training methods.

\section{Acknowledgements}

The authors would like to thank all subjects for their participation. The study was conducted without funding from companies or manufacturers.

\section{Conflict of Interest}

The authors declare that they have no conflict of interest.

\section{References}

[1] Markovic G. Does plyometric training improve vertical jump height? A meta-analytical review. Br J Sports Med 2007; 41: 349-355

[2] Knuttgen HG, Kraemer W]. Terminology and measurement in exercise performance. J Strength Cond Res 1987; 1: 1

[3] Markovic G, Mikulic P. Neuro-musculoskeletal and performance adaptations to lower-extremity plyometric training. Sports Med 2010; 40: 859-895

[4] Sheppard JM, Dingley AA, Janssen I et al. The effect of assisted jumping on vertical jump height in high-performance volleyball players. J Sci Med Sport 2011; 14: 85-89 
[5] Argus CK, Gill ND, Keogh JW et al. Kinetic and training comparisons between assisted, resisted, and free countermovement jumps. J Strength Cond Res 2011; 25: 2219-2227

[6] Markovic S, Mirkov DM, Knezevic OM et al. Jump training with different loads: Effects on jumping performance and power output. Eur J Appl Physiol 2013; 113: 2511-2521

[7] Markovic G, Vuk S, Jaric S. Effects of jump training with negative versus positive loading on jumping mechanics. Int J Sports Med 2011; 32 : 365-372

[8] Negra $\mathrm{Y}$, Chaabene $\mathrm{H}$, Sammoud S et al. The increased effectiveness of loaded versus unloaded plyometric-jump training in improving muscle power, speed, change-of-direction, and kicking-distance performance in prepubertal male soccer players. Int J Sports Physiol Perform 2019. doi:10.1123/ijspp.2018-0866 1-25

[9] Jimenez-Reyes P, Samozino P, Brughelli M et al. Effectiveness of an individualized training based on force-velocity profiling during jumping. Front Physiol 2017; 7: 677

[10] Rosas F, Ramirez-Campillo R, Diaz D et al. Jump training in youth soccer players: Effects of haltere type handheld loading. Int I Sports Med 2016; 37: 1060-1065

[11] Kobal R, Pereira LA, Zanetti V et al. Effects of unloaded vs. loaded plyometrics on speed and power performance of elite young soccer players. Front Physiol 2017; 8: 742

[12] Harriss DJ, MacSween A, Atkinson G. Standards for ethics in sport and exercise science researc: 2020 update. Int J Sports Med 2019; 40: 813-817
[13] Mangine GT, Hoffman JR, Gonzalez AM et al. The effect of training volume and intensity on improvements in muscular strength and size in resistance-trained men. Physiol Rep 2015; 3: e12472

[14] Bloomquist $\mathrm{K}$, Langberg $\mathrm{H}$, Karlsen $\mathrm{S}$ et al. Effect of range of motion in heavy load squatting on muscle and tendon adaptations. Eur J Appl Physiol 2013; 113: 2133-2142

[15] Linthorne NP. Analysis of standing vertical jumps using a force platform. Am J Phys 2001; 69: 1198-1204

[16] Cotterman ML, Darby LA, Skelly WA. Comparison of muscle force production using the Smith machine and free weights for bench press and squat exercises. J Strength Cond Res 2005; 19: 169-176

[17] Markovic G, Jaric S. Positive and negative loading and mechanical output in maximum vertical jumping. Med Sci Sports Exerc 2007; 39: 1757-1764

[18] Cohen J. Statistical Power for the Behavioral Sciences. Hillsdale, NJ: Lawrence Erlbaum Associates; 1988

[19] Behm DG, Sale DG. Intended rather than actual movement velocity determines velocity-specific training response. J Appl Physiol (1985) 1993; 74: 359-368

[20] Sale D, MacDougall D. Specificity in strength training: a review for the coach and athlete. Can J Appl Sport Sci 1981; 6: 87-92

[21] Srinivasan RC, Lungren MP, Langenderfer JE et al. Fiber type composition and maximum shortening velocity of muscles crossing the human shoulder. Clin Anat 2007; 20: 144-149

[22] Bogdanis GC, Tsoukos A, Brown LE et al. Muscle fiber and performance changes after fast eccentric complex training. Med Sci Sports Exerc 2018; 50: 729-738 